\title{
The association between cognitive impairment/dementia and albuminuria: a systematic review and meta-analysis
}

\author{
Hongqin $\mathrm{Li}^{1,2} \cdot$ Shuailin $\mathrm{Zhao}^{1} \cdot$ Ruiyu Wang ${ }^{1} \cdot$ Baoshan Gao ${ }^{1}$
}

Received: 16 April 2021 / Accepted: 15 August 2021 / Published online: 28 August 2021

(c) The Author(s) 2021

\begin{abstract}
Background To identify the association between albuminuria and dementia or cognitive impairment.

Methods The literature search was performed to identify relevant scientific studies through August 2019, including PubMed/ Medline and EMBASE. For inclusion, the studies had to fulfil the following criteria: population-based cohort, case-control or cross-sectional studies; quantifying an association of albuminuria with cognitive impairment or dementia; and reported odds ratio (OR), and the corresponding 95\% confidential interval (95\% CI). Random effects model was used to yield pooled estimates.

Results A total of 16 studies (11 cohort studies and five cross-sectional studies) were included in the meta-analyses. Based on the fully adjusted estimates, albuminuria was associated with a significant higher risk of cognitive impairment or dementia. Furthermore, the same trend existed for cognitive impairment and dementia, respectively. In addition, both of Alzheimer's diseases $(\mathrm{AD})$ and vascular dementia $(\mathrm{VaD})$ were significantly associated with albuminuria.

Conclusion Albuminuria was significantly associated with cognitive impairment and dementia. Corresponding to an earlier subclinical time-point in kidney disease progress, albuminuria may be a potential factor predicting the future occurrence of dementia.
\end{abstract}

Keywords Albuminuria $\cdot$ Cognitive impairment $\cdot$ Dementia $\cdot$ Meta-analysis

\section{Introduction}

There is an increasing number of people diagnosed with dementia worldwide, comprising major public health concerns [1]. Among individuals aged more than 60 years old, $5-7 \%$ of them are estimated to suffer from dementia, whereas the prevalence of mild cognitive impairment (MCI) is approximated to $20-30 \%[2,3]$. The identification of modifiable risk factors of cognitive decline or dementia is of vital importance to develop preventive strategies $[4,5]$.

As a marker of renal microvascular disease, albuminuria often occurs in the presence of hypertension and diabetes mellitus (DM), and shares common risk factors with dementia, including increasing age, elevated systolic blood

Baoshan Gao

gaobs@jlu.edu.cn

1 Department II of Urology, First Hospital of Jilin University, No. 71 Xinmin Street, Changchun 130021, Jilin, China

2 School of Pharmaceutical Science, Jilin University, Changchun, Jilin, China pressure (SBP), and increased levels of inflammation factors $[6,7]$. Previous studies has identified albuminuria as a potential risk factor for dementia and cognitive impairment [8-10]. Furthermore, Georgakis et al. had conducted a metaanalysis and identified that albuminuria was independently associated with cognitive impairment, dementia and cognitive decline in 2017 [11]. Adding newly reported data, this meta-analysis aimed to further clarify the association between albuminuria and dementia or cognitive impairment.

\section{Materials and methods}

\section{Literature search}

The literature search of computerized databases was performed to identify relevant scientific studies through August 2019, including PubMed/Medline and EMBASE. Two search themes were combined with the Boolean Operator "And". The first Boolean search used the terms of "albuminuria", "proteinuria" or "kidney disease", and the second 
theme was cognitive impairment using subject headings, including "dementia", "cognition", "cognitive impairment", or "Alzheimer's disease". In addition, the reference lists of identified studies were scanned to enhance the searches. This meta-analysis was designed, conducted and reported following the pre-determined protocol in accordance with the Meta-analysis of Observational Studies in Epidemiology (MOOSE) reporting guidelines [12].

\section{Study selection}

Hongqin Li and Baoshan Gao independently assessed the eligibility of each searching result. Full texts of selected articles were reviewed to further identify eligible studies included in the study, after screening all the potential articles with reading titles and abstracts. The disagreement was resolved by discussion. For inclusion, the study had to meet the following criteria: adult participants with aged $\geq 18$; longitudinal studies (cohort or case-control) or cross-sectional studies; quantifying an association of albuminuria with cognitive impairment or dementia; and reported odds ratio (OR), and the corresponding 95\% confidential interval (95\% CI). Studies with (1) involvement of solely children/adolescents and referring exclusively to hemodialysis patients, or patients with chronic autoimmune disorders or HIV-infected, (2) in vitro and animal studies, (3) reviews, case reports, abstracts, editorials, and comments would be excluded.

\section{Data extraction and quality assessment}

Shuailin Zhao and Ruiyu Wang extracted data from all eligible studies respectively, with any disagreement resolved by consensus. For each relevant study, the following data were extracted: the first author's last name, year of publication, study design, geographic location, sample size, median age, gender composition, clinical characteristics (body mass index [BMI], diabetes mellitus [DM], hypertension, cardiovascular disease [CVD], blood pressure [BP], albuminuria, estimated glomerular filtration rate [eGFR], cholesterol levels), exposures, outcome assessment, and analysis results. We adopted an evaluation system based on Newcastle-Ottawa scale (NOS) to assess the quality for both longitudinal and cross-sectional studies [13].

\section{Statistical analysis}

Albuminuria was defined by albumin-to-creatinine ratio (ACR) or 24-h urinary albumin excretion; cut-off points of either $(\geq 30 \mathrm{mg} / \mathrm{gCr}$ or $\mathrm{mg} / 24$-h, respectively), or (gender-specific $\geq 17$ in men and $\geq 25 \mathrm{mg} / \mathrm{gCr}$ or $\mathrm{mg} / 24$-h in women, respectively) were used [14]. Microalbuminuria was defined as excretion of $30-300 \mathrm{mg} / 24 \mathrm{~h}$ of albumin, whereas macroalbuminuria was defined as excretion of more than $300 \mathrm{mg} / 24 \mathrm{~h}$ of albumin.

Two major outcomes were examined, including dichotomized cognitive impairment/ dementia outcomes and continuous cognitive function measures. The first included cognitive impairment, defined by validated instruments, and dementia based on clinical diagnostic criteria. Various validated neuropsychological tests were used to evaluate continuous cognitive function. The diagnosis of dementia, and classification of Alzheimer's diseases (AD) and vascular dementia $(\mathrm{VaD})$ were based on clinical diagnostic criteria.

The ORs were used as the common measure of association, and both relative risks (RRs) and hazard ratios (HRs) were considered equivalent to ORs [15]. ORs and 95\% CIs for the effect of albuminuria on the risk of cognitive impairment and dementia were pooled, implemented with randomeffects models in meta-analyses. The heterogeneity among the studies was estimated with the $Q$ test and $I^{2}$ statistics [16]. Heterogeneity exists if $P$ value of $Q$ test $<0.05$. We had stratified the AD and $\mathrm{VaD}$ for further analysis. Subgroup analyses by study design were carried out, to assess the potential influence on the association. Publication bias was assessed through the Egger's test and funnel plots [17, 18]. All analyses were done with Stata software version 12 (StataCorp LP, College Station, TX, USA).

\section{Results}

\section{Literature search and study characteristics}

Our initial search yielded a total of 1106 unique citations, of which 36 articles were considered to be potentially relevant and identified to retrieval with full-text review (Fig. 1). Another 23 articles were further excluded for the following reasons: association not evaluated $(n=3)$; no ORs/HRs or the corresponding 95\% CI $(n=17)$; review or editorial $(n=2)$ and HIV patients $(n=1)$. Another three eligible studies were identified through references of relevant publications.

Finally, 16 studies (11 longitudinal and five cross-sectional studies), with a total of 127,296 participants (5488 cases of cognitive impairment, and 1266 cases of dementia) were included in the meta-analysis [19-34]. Among all the 16 studies, six studies explored the association with dementia and five of them made a stratification analysis on $A D$ and $\operatorname{VaD}[21,22,26,30-32]$. One study only contained the analysis with a continuous measure of cognitive impairment. The majority of studies provided results of multivariable analyses, adjusting major confounders, including age, sex, estimated glomerular filtration rate (eGFR), cardiovascular disease, diabetes, and hypertension. The average age for the participants ranged from 50 to 78 across all studies. Table 1 
Fig. 1 Flow diagram

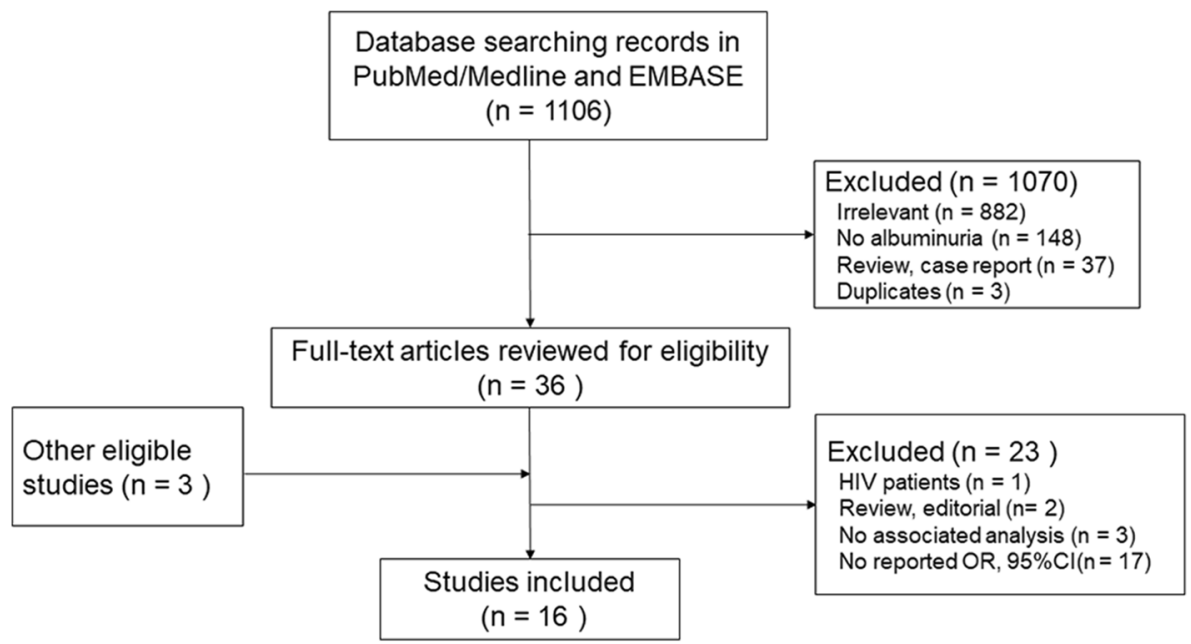

provides the detailed characteristics of all the included population-based studies, including the NOS scale.

\section{Meta-analysis}

Based on the fully adjusted estimates, albuminuria was associated with a significant higher risk of cognitive impairment or dementia (OR 1.20, 95 CI 1.12-1.28; $P<0.05$ ) as shown in Fig. 2. Furthermore, the same trend existed for cognitive impairment (OR 1.18, 95 CI 1.09-1.27; $P<0.05$ ) and dementia (OR 1.32, 95 CI 1.10-1.58; $P<0.05$ ), respectively. In addition, both of $\mathrm{AD}$ and $\mathrm{VaD}$ were significantly associated with albuminuria (Fig. 3, AD: OR 1.33, $95 \mathrm{CI}$ 1.06-1.67; $P<0.05$, and $\mathrm{VaD}$ : OR 2.32, 95 CI 1.59-3.38; $P<0.05)$. After excluding 5 cross-sectional studies, the pooled estimates for the remaining cohort studies revealed a significant association between albuminuria and cognitive impairment or dementia (Supplementary Fig. 1).

\section{Heterogeneity}

There was significant heterogeneity for all studies included in this meta-analysis $\left(I^{2}=61.5 \%, P<0.001\right)$. In addition, significant heterogeneity was identified for studies on cognitive impairment $\left(I^{2}=66.2 \%, P=0.001\right)$, whereas there was mild-to-moderate heterogeneity for studies with outcomes of dementia $\left(I^{2}=48.4 \%, P=0.085\right)$.

\section{Publications bias}

The funnel plot showed an obvious asymmetry (Fig. 4). And the Egger's test also showed evidence of publication bias $(P<0.01)$.

\section{Discussion}

This systematic review and meta-analysis suggested that individuals with albuminuria had significantly a higher risk of cognitive impairment or dementia. Stratifying by study design, the pooled results for all cohort studies revealed the same trend between albuminuria and risk of cognitive impairment or dementia.

Our findings were consistent with the previous metaanalysis that albuminuria was independently associated with cognitive impairment and dementia [11,35]. As an increasing prevalence of dementia worldwide, the identification of modifiable risk factors of cognitive decline or dementia is of vital importance. However, previous evidence had a high heterogeneity due to various measurements, different study design and statistical analysis. In this metaanalysis, we had a more restricted selection criteria, that only studies quantified an association between albuminuria and cognitive impairment or dementia and reported OR and 95\% CI were included to better evaluate the association. In addition, two studies based on large prospective registry, with a least of 10 years follow-up in Japanese and Norway population and stratified analysis of dementia subtypes, were added to update the evidence on albuminuria and cognitive impairment/dementia.

Although the mechanism underlying the relationship between albuminuria and dementia remained undermined, several plausible mechanisms may account for the association. First, proteinuria may act as a surrogate marker of oxidative stress, which plays an important role in the pathogenesis of dementia [36]. Paragh et al. found that defect in HDL-associated antioxidant capacity played a role in the pathogenesis of $\mathrm{AD}$ and $\mathrm{VaD}$ [37]. In addition, the process of atherosclerosis, often developing for several decades due to endothelial damage, may contribute to neurodegeneration related to $\mathrm{AD}$ [38]. Besides, atherosclerosis associated 


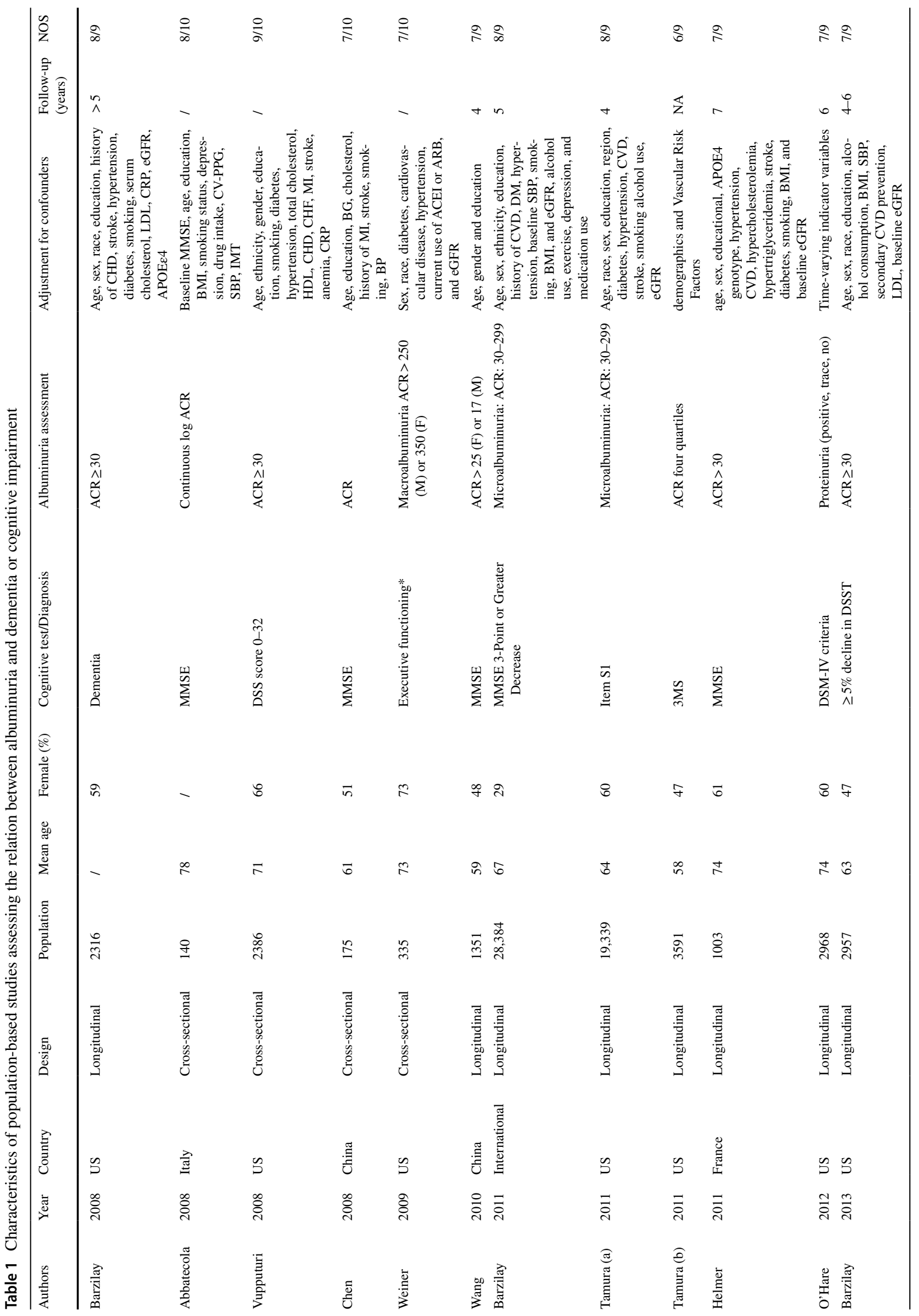




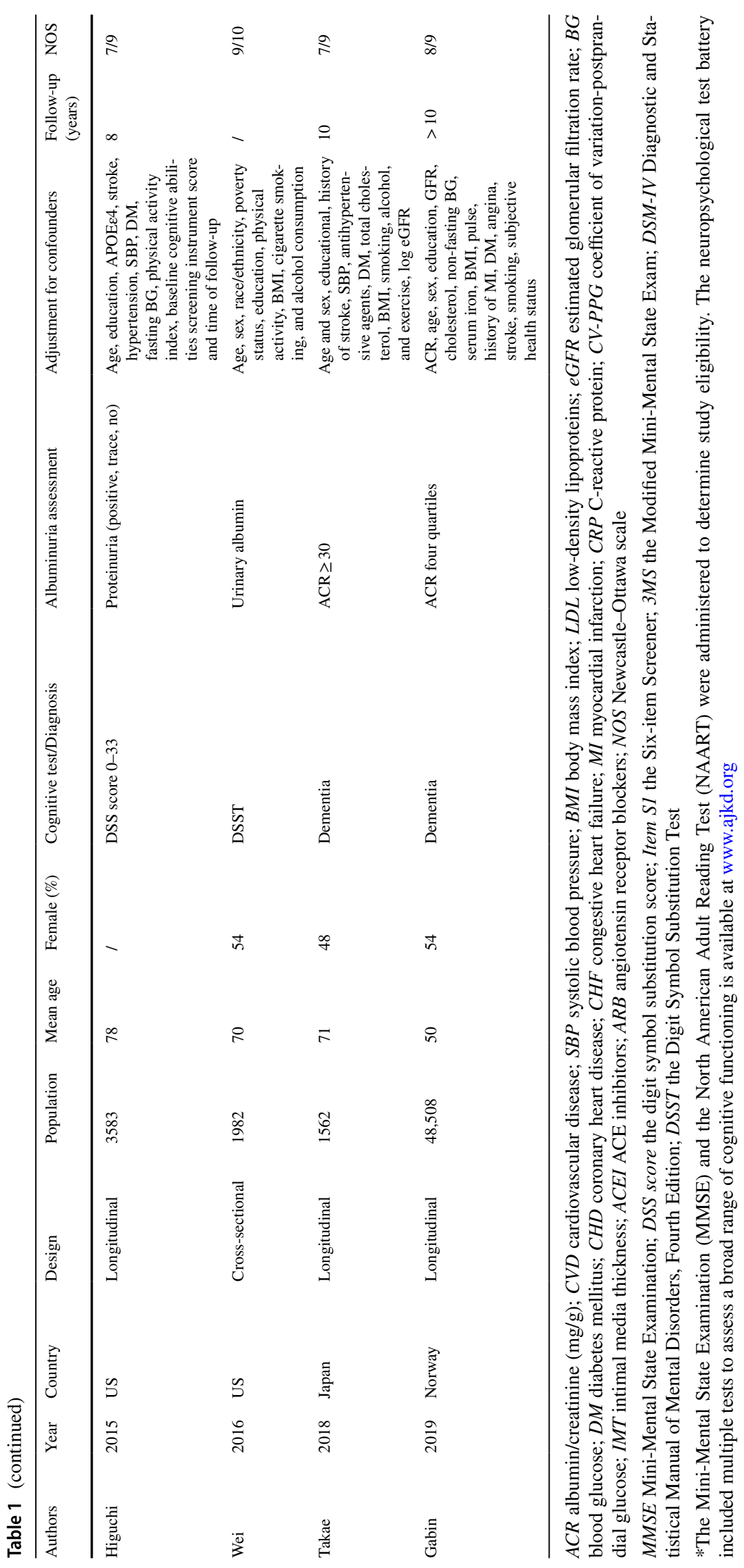




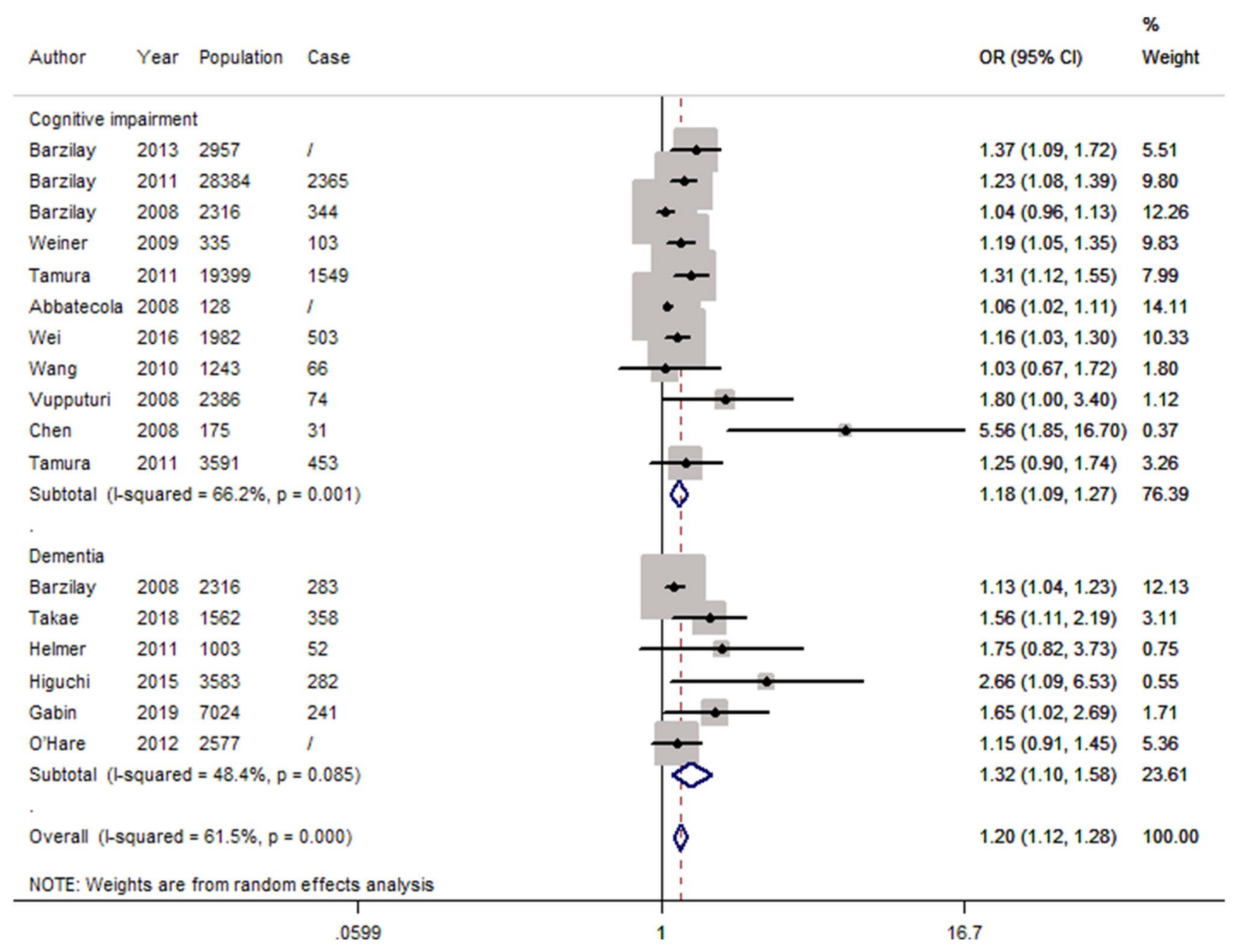

Fig. 2 Forest plot of population-based studies assessing the relation between albuminuria and dementia or cognitive impairment (fully adjusted estimates)

diseases, including hypertension and DM were proven to be associated with poor performance on cognitive function test, and an increased risk of dementia [39, 40]. Furthermore, microvascular pathology, correlated with increased risks of various vascular diseases in kidney, heart and brain, may participate in the pathogenesis of $\mathrm{VaD}$, and possibly AD. Autopsy data showed that microvascular pathology was associated with not only $\mathrm{VaD}$, but also $\mathrm{AD}$ and all-cause dementia [41].

The association of albuminuria and dementia probably indicates concurrent pathology in kidneys and brain. Although the mechanism is not fully understood, evidence has proven the association of chronic kidney disease and cognitive dysfunction [42, 43]. However, those studies mainly focused on advanced kidney damage stages [44, 45]. Microalbuminuria, corresponding to an earlier subclinical time-point in kidney disease progress, could work as a more sensitive marker.
As an important method to reveal risk trends, metaanalysis provides a more precise risk estimates. In addition, most of the studies included in our meta-analysis adopted a cohort design, and the estimates with cohort studies separately were calculated, which might reduce the possibility of recall and selection bias. Moreover, the included studies contained various ethnicity, which might increase the generalizability of the findings. However, several limitations of this meta-analysis should be acknowledged. First, since only observational studies were obtained, there was a possibility that other factors might account for the association. In addition, compared with studies on dementia, the heterogeneity for studies on cognitive impairment was significant. Several methodologic issues might contribute to the substantial heterogeneity observed in the meta-analyses for cognitive impairment populations, including: (1) different criteria to measure albuminuria; (2) variation in study population 


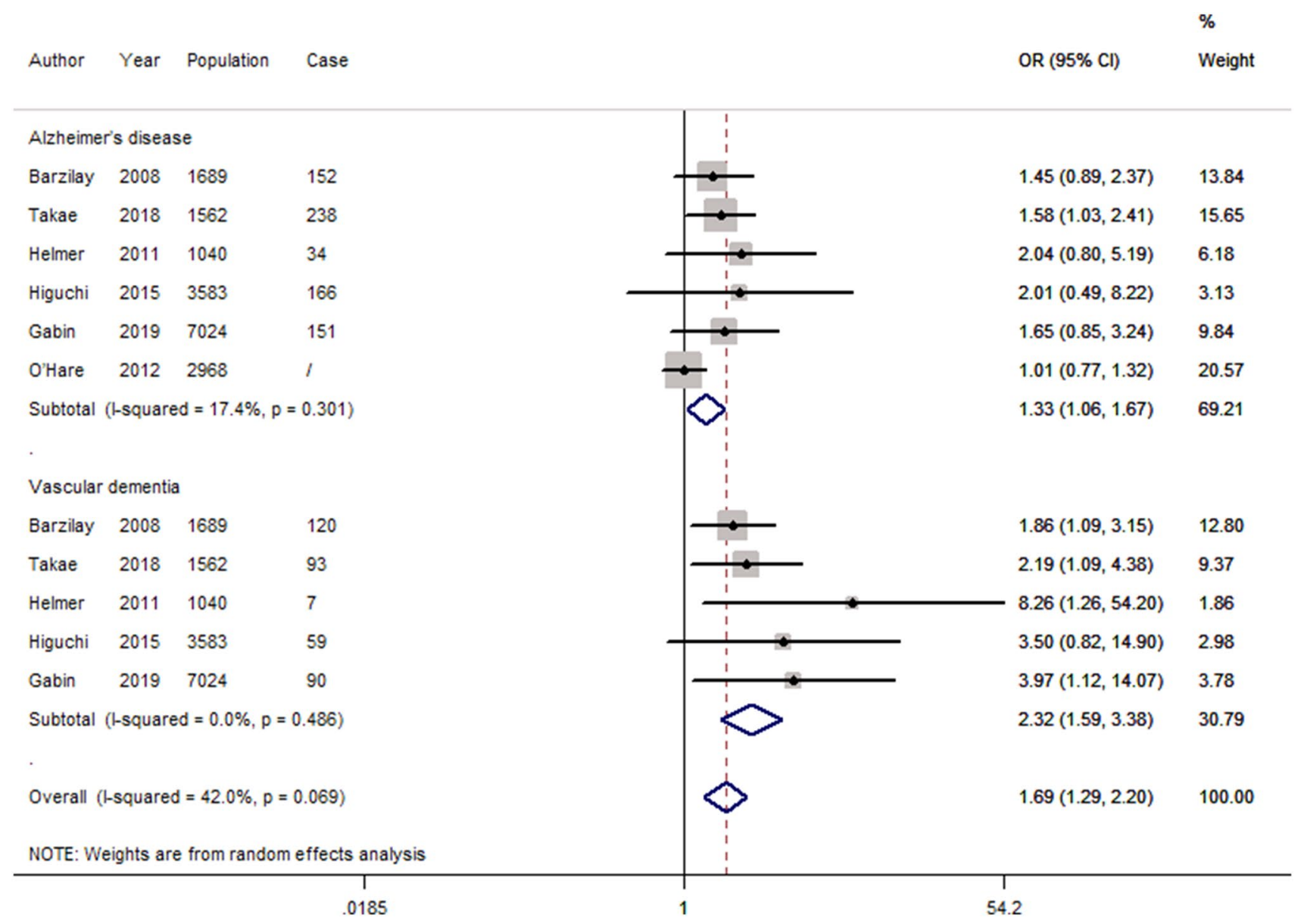

Fig. 3 Forest plot of studies assessing the relation between albuminuria and dementia, stratified by AD and PD (fully adjusted estimates)

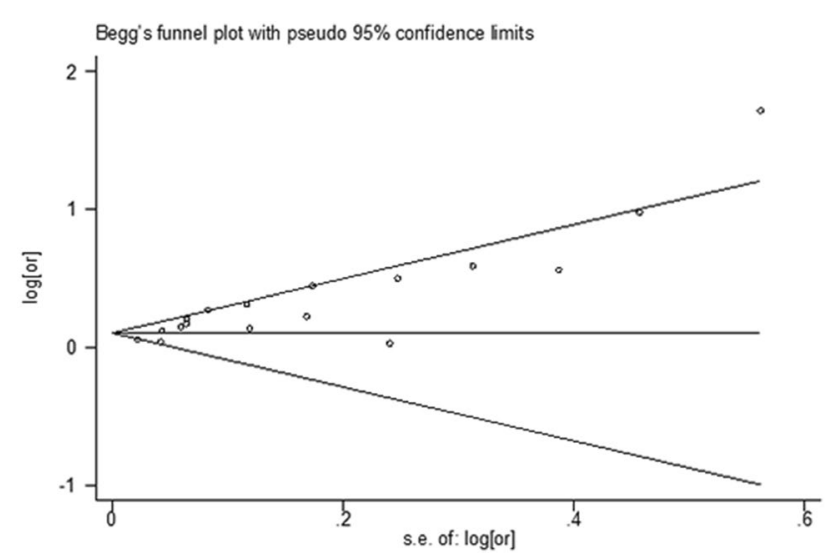

Fig. 4 Funnel plot

(e.g. ethnicity, age range, concurrent medication, and comorbidity); (3) adjusting different confounders; and (4) different assessment tools of cognitive functioning, and one study only having continuous measures of cognition. Last, the results of the meta-analysis should be take cautiously since significant publication bias was revealed and the direct exchange of HR/RR to OR. Further evidence are warranted to verify our findings.

In summary, albuminuria was significantly associated with cognitive impairment and dementia. Corresponding to an earlier subclinical time-point in kidney disease progress, albuminuria may be a potential factor predicting the future occurrence of dementia. Targeting the pathogenesis of albuminuria or treating modifiable risk factors may have clinical implications to prevent dementia, or delay disease progression.

Supplementary Information The online version contains supplementary material available at https://doi.org/10.1007/s10157-021-02127-3.

Author contributions $\mathrm{HL}$ and BG carried out the studies, participated in collecting data, and drafted the manuscript. SZ and RW performed the statistical analysis and participated in its design. All authors read and approved the final manuscript.

Funding The study supported by Science and technology project of Jilin Provincial Department of Education (No.JJKH20180100KJ) and National Natural Science Foundation of China (No.81873873). 
Availability of data and materials The datasets used and/or analyzed during the current study are available from the corresponding author on reasonable request.

\section{Declarations}

Conflict of interest All the authors declare that they have no conflict of interest.

Ethic statement This study was meta-analysis, so ethic approval was not required.

Open Access This article is licensed under a Creative Commons Attribution 4.0 International License, which permits use, sharing, adaptation, distribution and reproduction in any medium or format, as long as you give appropriate credit to the original author(s) and the source, provide a link to the Creative Commons licence, and indicate if changes were made. The images or other third party material in this article are included in the article's Creative Commons licence, unless indicated otherwise in a credit line to the material. If material is not included in the article's Creative Commons licence and your intended use is not permitted by statutory regulation or exceeds the permitted use, you will need to obtain permission directly from the copyright holder. To view a copy of this licence, visit http://creativecommons.org/licenses/by/4.0/.

\section{References}

1. Ferri CP, Prince M, Brayne C, Brodaty H, Fratiglioni L, Ganguli M, et al. Global prevalence of dementia: a Delphi consensus study. Lancet. 2005;366(9503):2112-7. https://doi.org/10.1016/S01406736(05)67889-0.

2. Prince M, Bryce R, Albanese E, Wimo A, Ribeiro W, Ferri CP. The global prevalence of dementia: a systematic review and metaanalysis. Alzheimers Dement. 2013;9(1):63-75.e2. https:// doi.org/10.1016/j.jalz.2012.11.007.

3. Ward A, Arrighi HM, Michels S, Cedarbaum JM. Mild cognitive impairment: disparity of incidence and prevalence estimates. Alzheimers Dement. 2012;8(1):14-21. https://doi.org/10.1016/j. jalz.2011.01.002.

4. Plassman BL, Williams JW Jr, Burke JR, Holsinger T, Benjamin S. Systematic review: factors associated with risk for and possible prevention of cognitive decline in later life. Ann Intern Med. 2010;153(3):182-93. https://doi.org/10.7326/0003-4819-153-3201008030-00258.

5. Norton S, Matthews FE, Barnes DE, Yaffe K, Brayne C. Potential for primary prevention of Alzheimer's disease: an analysis of population-based data. Lancet Neurol. 2014;13(8):788-94. https:// doi.org/10.1016/S1474-4422(14)70136-X.

6. de la Torre JC. Alzheimer disease as a vascular disorder: nosological evidence. Stroke. 2002;33(4):1152-62. https://doi.org/10. 1161/01.str.0000014421.15948.67.

7. Barzilay JI, Peterson D, Cushman M, Heckbert SR, Cao JJ, Blaum $\mathrm{C}$, et al. The relationship of cardiovascular risk factors to microalbuminuria in older adults with or without diabetes mellitus or hypertension: the cardiovascular health study. Am J Kidney Dis. 2004;44(1):25-34. https://doi.org/10.1053/j.ajkd.2004.03.022.

8. Deckers K, van Boxtel MP, Schiepers OJ, de Vugt M, Munoz Sanchez JL, Anstey KJ, et al. Target risk factors for dementia prevention: a systematic review and Delphi consensus study on the evidence from observational studies. Int J Geriatr Psychiatry. 2015;30(3):234-46. https://doi.org/10.1002/gps.4245.
9. Madero M, Gul A, Sarnak MJ. Cognitive function in chronic kidney disease. Semin Dial. 2008;21(1):29-37. https://doi.org/10. 1111/j.1525-139X.2007.00384.x.

10. Murray AM. Cognitive impairment in the aging dialysis and chronic kidney disease populations: an occult burden. Adv Chronic Kidney Dis. 2008;15(2):123-32. https://doi.org/10. 1053/j.ackd.2008.01.010.

11. Georgakis MK, Dimitriou NG, Karalexi MA, Mihas C, Nasothimiou EG, Tousoulis D, et al. Albuminuria in association with cognitive function and dementia: a systematic review and metaanalysis. J Am Geriatr Soc. 2017;65(6):1190-8. https://doi.org/ 10.1111 /jgs. 14750 .

12. Stroup DF, Berlin JA, Morton SC, Olkin I, Williamson GD, Rennie D, et al. Meta-analysis of observational studies in epidemiology: a proposal for reporting. Meta-analysis Of Observational Studies in Epidemiology (MOOSE) group. JAMA. 2000;283(15):2008-12. https://doi.org/10.1001/jama.283.15. 2008.

13. Stang A. Critical evaluation of the Newcastle-Ottawa scale for the assessment of the quality of nonrandomized studies in metaanalyses. Eur J Epidemiol. 2010;25:603-5.

14. Crowe E, Halpin D, Stevens P, Guideline DG. Early identification and management of chronic kidney disease: summary of NICE guidance. BMJ. 2008;337: a1530. https://doi.org/10.1136/bmj. a1530.

15. Zhang J, Yu KF. What's the relative risk? A method of correcting the odds ratio in cohort studies of common outcomes. JAMA. 1998;280(19):1690-1. https://doi.org/10.1001/jama.280.19.1690.

16. Higgins JP, Thompson SG. Quantifying heterogeneity in a metaanalysis. Stat Med. 2002;21(11):1539-58. https://doi.org/10.1002/ sim. 1186 .

17. Egger M, Davey Smith G, Schneider M, Minder C. Bias in meta-analysis detected by a simple, graphical test. BMJ. 1997;315(7109):629-34. https://doi.org/10.1136/bmj.315.7109. 629.

18. Begg CB, Mazumdar M. Operating characteristics of a rank correlation test for publication bias. Biometrics. 1994;50(4):1088-101.

19. Barzilay JI, Lovato JF, Murray AM, Williamson J, Ismail-Beigi F, Karl D, et al. Albuminuria and cognitive decline in people with diabetes and normal renal function. Clin J Am Soc Nephrol. 2013;8(11):1907-14. https://doi.org/10.2215/cjn.11321112.

20. Barzilay JI, Gao P, O’ Donnell M, Mann JF, Anderson C, Fagard R, et al. Albuminuria and decline in cognitive function: the ONTARGET/TRANSCEND studies. Arch Intern Med. 2011;171(2):142_50. https://doi.org/10.1001/archinternmed.2010.502.

21. Barzilay JI, Fitzpatrick AL, Luchsinger J, Yasar S, Bernick C, Jenny NS, et al. Albuminuria and dementia in the elderly: a community study. Am J Kidney Dis. 2008;52(2):216-26. https://doi. org/10.1053/j.ajkd.2007.12.044.

22. Takae K, Hata J, Ohara T, Yoshida D, Shibata M, Mukai N, et al. Albuminuria increases the risks for both Alzheimer disease and vascular dementia in community-dwelling Japanese elderly: the Hisayama study. J Am Heart Assoc. 2018. https://doi.org/10.1161/ jaha.117.006693

23. Weiner DE, Bartolomei K, Scott T, Price LL, Griffith JL, Rosenberg I, et al. Albuminuria, cognitive functioning, and white matter hyperintensities in homebound elders. Am J Kidney Dis. 2009;53(3):438-47. https://doi.org/10.1053/j.ajkd.2008.08.022.

24. Kurella Tamura M, Muntner P, Wadley V, Cushman M, Zakai NA, Bradbury BD, et al. Albuminuria, kidney function, and the incidence of cognitive impairment among adults in the United States. Am J Kidney Dis. 2011;58(5):756-63. https://doi.org/10. 1053/j.ajkd.2011.05.027.

25. Abbatecola AM, Barbieri M, Rizzo MR, Grella R, Laieta MT, Quaranta E, et al. Arterial stiffness and cognition in elderly persons with impaired glucose tolerance and microalbuminuria. J 
Gerontol A Biol Sci Med Sci. 2008;63(9):991-6. https://doi.org/ 10.1093/gerona/63.9.991.

26. Helmer C, Stengel B, Metzger M, Froissart M, Massy ZA, Tzourio $\mathrm{C}$, et al. Chronic kidney disease, cognitive decline, and incident dementia: the 3C Study. Neurology. 2011;77(23):2043-51. https:// doi.org/10.1212/WNL.0b013e31823b4765.

27. Wei Y, Wei YK, Zhu J. Early markers of kidney dysfunction and cognitive impairment among older adults. J Neurol Sci. 2017;375:209-14. https://doi.org/10.1016/j.jns.2017.01.071.

28. Wang F, Zhang L, Liu L, Wang H. Level of kidney function correlates with cognitive decline. Am J Nephrol. 2010;32(2):117-21. https://doi.org/10.1159/000315618.

29. Vupputuri S, Shoham DA, Hogan SL, Kshirsagar AV. Microalbuminuria, peripheral artery disease, and cognitive function. Kidney Int. 2008;73(3):341-6. https://doi.org/10.1038/sj.ki.5002672.

30. Higuchi M, Chen R, Abbott RD, Bell C, Launer L, Ross GW, et al. Mid-life proteinuria and late-life cognitive function and dementia in elderly men: the Honolulu-Asia Aging Study. Alzheimer Dis Assoc Disord. 2015;29(3):200-5. https://doi.org/10.1097/wad. 0000000000000082.

31. Gabin JM, Romundstad S, Saltvedt I, Holmen J. Moderately increased albuminuria, chronic kidney disease and incident dementia: the HUNT study. BMC Nephrol. 2019;20(1):261. https://doi.org/10.1186/s12882-019-1425-8.

32. O'Hare AM, Walker R, Haneuse S, Crane PK, McCormick WC, Bowen JD, et al. Relationship between longitudinal measures of renal function and onset of dementia in a community cohort of older adults. J Am Geriatr Soc. 2012;60(12):2215-22. https://doi. org/10.1111/j.1532-5415.2012.04238.x.

33. Chen XP, Wu J, Peng Q, Huang H, Mao ZX, Huang Y, et al. Relationship between microalbuminuria and cognition in primary hypertension patients. Zhonghua Xin Xue Guan Bing Za Zhi. 2008;36(8):722-5.

34. Kurella Tamura M, Xie D, Yaffe K, Cohen DL, Teal V, Kasner SE, et al. Vascular risk factors and cognitive impairment in chronic kidney disease: the Chronic Renal Insufficiency Cohort (CRIC) study. Clin J Am Soc Nephrol. 2011;6(2):248-56. https://doi.org/ 10.2215/CJN.02660310.

35. Deckers K, Camerino I, van Boxtel MP, Verhey FR, Irving K, Brayne $\mathrm{C}$, et al. Dementia risk in renal dysfunction: a systematic review and meta-analysis of prospective studies. Neurology. 2017;88(2):198-208. https://doi.org/10.1212/WNL.0000000000 003482 .

36. Deschamps V, Barberger-Gateau P, Peuchant E, Orgogozo JM. Nutritional factors in cerebral aging and dementia: epidemiological arguments for a role of oxidative stress. Neuroepidemiology. 2001;20(1):7-15. https://doi.org/10.1159/000054752.
37. Paragh G, Balla P, Katona E, Seres I, Egerhazi A, Degrell I. Serum paraoxonase activity changes in patients with Alzheimer's disease and vascular dementia. Eur Arch Psychiatry Clin Neurosci. 2002;252(2):63-7. https://doi.org/10.1007/s004060200013.

38. Hofman A, Ott A, Breteler MM, Bots ML, Slooter AJ, van Harskamp F, et al. Atherosclerosis, apolipoprotein E, and prevalence of dementia and Alzheimer's disease in the Rotterdam Study. Lancet. 1997;349(9046):151-4. https://doi.org/10.1016/S01406736(96)09328-2.

39. Launer LJ, Masaki K, Petrovitch H, Foley D, Havlik RJ. The association between midlife blood pressure levels and late-life cognitive function. The Honolulu-Asia Aging Study. JAMA. 1995;274(23):1846-51.

40. Launer LJ, Ross GW, Petrovitch H, Masaki K, Foley D, White LR, et al. Midlife blood pressure and dementia: the Honolulu-Asia aging study. Neurobiol Aging. 2000;21(1):49-55. https://doi.org/ 10.1016/s0197-4580(00)00096-8.

41. White L, Petrovitch H, Hardman J, Nelson J, Davis DG, Ross $\mathrm{GW}$, et al. Cerebrovascular pathology and dementia in autopsied Honolulu-Asia Aging Study participants. Ann N Y Acad Sci. 2002;977:9-23. https://doi.org/10.1111/j.1749-6632.2002.tb047 94.x.

42. Etgen T, Chonchol M, Forstl H, Sander D. Chronic kidney disease and cognitive impairment: a systematic review and meta-analysis. Am J Nephrol. 2012;35(5):474-82. https://doi.org/10.1159/00033 8135.

43. Shen Z, Ruan Q, Yu Z, Sun Z. Chronic kidney disease-related physical frailty and cognitive impairment: a systemic review. Geriatr Gerontol Int. 2017;17(4):529-44. https://doi.org/10.1111/ggi. 12758.

44. Toyoda K, Ninomiya T. Stroke and cerebrovascular diseases in patients with chronic kidney disease. Lancet Neurol. 2014;13(8):823-33. https://doi.org/10.1016/S1474-4422(14) 70026-2.

45. Vogels SC, Emmelot-Vonk MH, Verhaar HJ, Koek HL. The association of chronic kidney disease with brain lesions on MRI or CT: a systematic review. Maturitas. 2012;71(4):331-6. https://doi.org/ 10.1016/j.maturitas.2012.01.008.

Publisher's Note Springer Nature remains neutral with regard to jurisdictional claims in published maps and institutional affiliations. 